\title{
Caracterización del estado nutricional y las condiciones sociales existente en las familias de la micro cuenca de Moyua, ciudad Darío Matagalpa. Nicaragua, 2015
}

\author{
Evelyn Calvo Reyes ${ }^{1}$ \\ Marcia Cordero Rizo ${ }^{2}$ \\ Yesica Ninoska Gómez ${ }^{3}$ \\ Waldin Omar Valdivia ${ }^{4}$
}

\begin{abstract}
El estudio se realizó en cuatro comunidades rurales del municipio de Ciudad Darío Matagalpa: Moyúa, Playitas, Puertas viejas, Papayal, pertenecen al micro cuenca del rio grande de Matagalpa, Nicaragua. Con el objetivo de caracterizar el estado nutricional y las condiciones sociales existentes en las familias. La metodología utilizada fue bajo la perspectiva del enfoque cuantitativo con componentes cualitativos, de corte transversal utilizando, la estadística descriptiva, con una población de 84 familias y una muestra 42 que representa el 50\% del total, se tomó de manera azar izada con los criterios: que en las familias existan por lo menos un niño o niña menor de cinco años de edad, tiempo de vivir en la comunidad de 6 años, las madres estuvieran anuentes a brindar la información. Los resultados obtenidos establecen que en las cuatro comunidades prevalece que la edad promedio de que las madres en el embarazo son 20 años y mayores de 30 en menor porcentaje, las madres realizan los controles prenatales mensualmente en su mayoría. El IMC en los infantes prevaleciendo posible riesgo de sobre peso, emaciado y obeso, las madres proporcionan leche materna a los infantes 2 a 24 meses de edad y lo sugerido por el ministerio de salud de 3- 6 meses lo que le proporciona minerales y vitaminas necesarias para su crecimiento. Las familias poseen servicios básicos desde energía eléctrica, agua potable y saneamiento, el material del piso, techo y paredes de las viviendas sobresale de tierra, zinc y paredes de ladrillos.
\end{abstract}

Palabras claves: Lactancia, Hábitos alimenticios, Índice de masa corporal (IMC), vivienda.

Recibido: 19 de septiembre de 2016

Aceptado: 28 de octubre de 2016

1 Docente Investigadora de la Facultad Multidisciplinaria, UNAN-Managua, FAREM-Matagalpa. Correo electrónico: emilyareyes@yahoo.es

2 Docente Investigadora de la Facultad Multidisciplinaria, UNAN-Managua, FAREM-Matagalpa. Correo electrónico: corderorizo@hotmail.com

3 Egresada de la carrera de Ingeniería Agronómica, UNAN-Managua, FAREM-Matagalpa. Correo electrónico: yesicaninoskag@gmail.com

4 Egresado de la carrera de Ingeniería Agronómica, UNAN-Managua, FAREM-Matagalpa. Correo electrónico: valdivia16@yahoo.com 


\title{
Characterization of the nutritional status and the current conditions among families of the micro watershed of Moyua- Darío, Matagalpa. Nicaragua, 2015
}

\begin{abstract}
The study was conducted in four rural communities of the municipality of Ciudad Darío, Matagalpa: Moyúa, Playitas, Puertas viejas, Papayal. All of those belong to the micro watershed of Río Grande of Matagalpa, Nicaragua. The study aimed to characterize the nutritional status and the current social conditions of the families. The methodology used followed the quantitative research design, and featured some qualitative elements. The population consisted of 84 families and the sample consisted of 42 , which represents to the $50 \%$ of the total population. The sample was randomly chosen and the following criteria were set: that the family had at least one child less than five years old, that the family had lived in the community for at least six years, and that the mothers were willing to give relevant information. The main research results reveal that 20-30 years of the average age of pregnancy. Moreover, they indicate that the mothers in the community annually take some protections measures. Children face a high risk of obesity and overweight. Mothers feed their babies from 2-24 months old; however, the Health Ministry suggests 3-6 months so as babies get the minerals and vitamins they need at an early age. The families have the basic services, such as electric energy, drinking water, floor, roof and brick walls.
\end{abstract}

Key words: Eating habits, body mass number, household. 


\section{INTRODUCCIÓN}

De acuerdo al informe de la Organización de las Naciones Unidas para la Agricultura y la Alimentación FAO,(2006) Todas estas transformaciones repercuten de manera inconsciente y negativa en la Seguridad Alimentaria de la población mundial, porque por un lado están aquellos que presentan problemas de hambre y desnutrición, y por otro aquellos que presentan enfermedades crónicas debido a la ingesta no adecuada y poco nutritiva de alimentos, a pesar de tener acceso físico a los mismos; no estando Nicaragua exenta de este fenómeno.El estado nutricional es uno de los componentes más importantes del estado de la salud de un individuo, ya que cuando este se encuentra alterado, afecta el rendimiento físico, mental y social. (Casanueva, et al., 2002).

Existe relación entre la pobreza y la inseguridad alimentaria nutricional, determinado por el (CEPAL, 1979) que define la pobreza como un síndrome situacional en el que se asocian el infra consumo, la desnutrición, la precariedad, condiciones de vivienda, los bajos niveles de educación, malas condiciones sanitarias, una inserción inestable en el aparato productivo o dentro de los estratos primitivos del mismo, actitudes de desaliento y anomia, poca participación en los mecanismos de integración social.

El artículo está orientado a caracterizar el estado nutricional y las condiciones de vida de las familias de la Micro Cuenca de Moyúa -Ciudad Darío, Matagalpa 2015, con el objetivo de determinar el estado nutricional de los niños y niñas menores de cinco años de edad a partir de los indicadores: conducta alimentaria, hábitos de consumo, índice de masa corporal, desnutrición materna, edad de la madre en el embarazo, tipo de embarazo, el historial clínico del infante como: peso al nacer del infante, peso, talla, IMC, duración de la lactancia materna y las condiciones de vida de las familias se mido a partir de los indicadores sociales como son: educación, composición familiar, distribución de los roles, calidad de la vivienda, disponibilidad económica familiar.

\section{MARCO CONCEPTUAL DE LA SEGURIDAD ALIMENTARIA NUTRICIONAL}

Ante de determinar el concepto de seguridad alimentaria nutricional es necesario considerar que en la Asamblea nacional de la Republica de Nicaragua presenta a la Ciudadanía la Ley $N^{\circ} .693$ "Ley de soberanía y Seguridad Alimentaria y Nutricional" (S.S.A.N), aprobada en este Poder del Estado el pasado 18 de Julio del 2009, publicado en la Gaceta Diario Oficial el 16 de Julio del mismo año.

La Ley tiene por objetivo garantizar el derecho de todas y todos los nicaragüense de contar con los alimentos suficientes, inóculos y nutritivos acordes a sus necesidades vitales que estos sean accesibles física, económica, social y culturalmente de forma oportuna y permanentemente, asegurando la disponibilidad, estabilidad y suficiencia de los mismos a través del desarrollo y rectoría por parte del estado de políticas públicas vinculadas a la soberanía y Seguridad Alimentaria Nutricional. El artículo 3 de la ley 693 Principios de la ley de Soberanía y Seguridad Alimentaria y Nutricional .los siguientes:

a. Disponibilidad: En virtud de este principio, el Estado promoverá la existencia de los recursos necesarios en el país para garantizar de manera permanente la estabilidad de la oferta de alimentos en cantidad y calidad suficientes, que permitan satisfacer las necesidades de alimentación y nutrición de la población.

b. Equidad y Acceso: Por este principio los programas económicos y sociales de las instituciones del Gobierno promoverán el desarrollo de las poblaciones LEY DE SOBERANÍA Y SEGURIDAD ALIMENTARIA Y NUTRICIONAL 21 con mayor índice de pobreza, tomando medidas que permitan obtener 
recursos para producir, acceder y/o disponer de alimentos. Así mismo propiciar medidas para que, en especial las mujeres productoras de alimentos, tengan acceso a los recursos técnicos y financieros, así como a bienes y servicios disponibles.

c. Consumo: Por este principio el Estado promueve la ingesta de los alimentos sanos e inocuos que se precisan en cantidad y calidad necesarias para que las personas tengan una alimentación adecuada y saludable. d. Utilización Biológica. Por este principio el Estado promoverá que se dé el máximo aprovechamiento que da el organismo de las personas a los nutrientes LEY DE SOBERANÍA Y SEGURIDAD ALIMENTARIA Y NUTRICIONAL 22 contenidos en los alimentos que consume, el mejoramiento de la salud de las personas y del entorno ambiental, genético e inmunológico.

d. Utilización Biológica: Por este principio el Estado promoverá que se dé el máximo aprovechamiento que da el organismos de las personas a los nutrientes contenidos en los alimentos que consume el mejoramiento de la salud de las personas y en el entorno ambiental, genético, e inmunológico.

Este articulo determina que todo ciudadano, tiene derecho a una alimentación saludable, es decir proporcionar todos los minerales, vitaminas, proteínas que el cuerpo necesita para su desarrollo embrionario e infantil.

\section{CONCEPTO DE SEGURIDAD ALIMENTARIA}

(FAO, 2007) plantea:" Existe seguridad alimentaria cuando todas las personas tienen en todo momento acceso físico y económico a suficientes alimentos inocuos y nutritivos para satisfacer sus necesidades alimentarias".

Mientras que para Bernal, (2010) en el estudio de la Inseguridad Alimentaria y hambre en niños en Venezuela: Diseño y validación de instrumentos, en niños padecen directamente las consecuencias de la falta de accesos a los alimentos y generalmente no o independencia necesarios para el óptimo crecimiento, el instrumento desarrollo, medición practica que permite conocer los avances o retrocesos del problema en la población vulnerable.

Ambos autores establecen que la inseguridad alimentaria está relacionada con la accesibilidad de los alimentos y estos a su vez contribuyen al desarrollo físico e intelectual de los individuos.

\section{Nutrición}

La nutrición se define como un conjunto de las funciones orgánicas de transformación y utilización de los alimentos para el crecimiento y la actividad de un ser vivo proporcionan energía para mantener la respiración y la vida de nuestro organismo, para mantener la temperatura corporal, posibilitar el movimiento, el crecimiento normal y la reparación de los tejidos (FAO, 2004:24).

\section{Desnutrición}

Para Delgado, (2005) la desnutrición representa un círculo vicioso y se presenta cuando una mujer sufre de desnutrición o no recibe una adecuada alimentación y un cuido durante el periodo de embarazo, tendrá un hijo desnutrido, mientras la madre recibe buena alimentación y control prenatal durante los meses de embarazo, tendrá un hijo sano que crecerá e ira a la escuela y tendrá hijo sanos.

Según la Encuesta Nicaragüense de Demografía y Salud ([ENDESA], 2006:07) se encontró que el 29\% de los niños menores de cinco años presentaba síntomas generales de infecciones Respiratorias Agudas (IRA) en Nicaragua.

Mientras que Montoya plantea que existe una gran relación entre la pobreza y la presencia de enfermedades y son un ciclo vicioso donde el centro es la malnutrición, enfermedad provocada por la pobreza e incidida por 
los factores externos como son .Situación económica, políticas públicas, intervenciones en nutrición y salud y sociales, que están ejerciendo en los hogares familiares y el país.

Lo cual coincide con (Mc Lachlan, 2006) establece que una buena nutrición o alimentación de los niños es uno de los factores biológicos más importante en el desarrollo cerebral y cognitivo, mismo que repercutirán durante toda la vida".

\section{Factores que indicen en la seguridad alimentaria nutricional}

Según la (OMS, 1997) "Los factores ambientales, incluyendo las enfermedades infecciosas, una dieta inadecuada o insegura y las consecuencias de la pobreza, son más importantes que la predisposición genética a la hora de producirse desviaciones de los valores de referencia del crecimiento"

Para valorar el estado nutricional, es fundamental estudiar el crecimiento. Hay evidencias de la relación entre el crecimiento insuficiente y el retraso del desarrollo mental, así como la asociación con el fracaso escolar y con la reducción de la capacidad intelectual (de Onís et al, 2000; OPS, 1998).

\section{Índice de Masa Corporal (IMC):}

La OMS (2003), el Índice de Masa Corporal es un indicador que es especialmente útil cuando se examina sobrepeso u obesidad. El IMC no aumenta con la edad como se observa con el peso y la talla. Un bebe por medio de su crecimiento, ganan peso relativamente rápido en comparación con su longitud en los primeros 6 meses de vida. El IMC baja posteriormente en la infancia y se mantiene relativamente estable desde la edad de dos (2) a los cinco (5) años.

Un IMC por debajo de 18,5 indica desnutrición o algún problema de salud, mientras que un IMC superior a 25 indica sobrepeso. Por encima de 30 hay obesidad leve, y por encima de 40 hay obesidad elevada que necesita seguimiento médico continuo. El índice ideal, por tanto, se sitúa entre los 20 y $25 \mathrm{Kg} / \mathrm{m} 2$ (Andreu, 2012).

Ecuación de IMC según, Andreu, (2012).

$$
\mathrm{IMC}=\frac{\operatorname{peso}(\mathrm{kg})}{\operatorname{altura}^{2}(\mathrm{~m})}
$$

Peso al nacer Según OMS (1979), “cuando hay bajo peso al nacer en los niños (menos de 2500 gramos), en las primeras horas de vida se debe a":

- Ingesta insuficiente de alimentos

- Infecciones frecuentes

- Enfermedades crónicas (VIH, Tuberculosis, cardiópatas).

- Evaluación del estado nutricional del adulto (madre de familia)

La Organización Mundial de la Salud (OMS, 2003) declara que la lactancia materna exclusiva reúne todos los requerimientos nutricionales hasta los 6 meses, con excepción de la vitamina $\mathrm{D}$ y de hierro en los lactantes prematuros y con bajo peso de nacimiento. Se recomienda como imprescindible la lactancia materna exclusiva durante los seis primeros meses del recién nacido. También recomiendan seguir amamantando a partir de los seis meses, al mismo tiempo que se va ofreciendo al bebé otros alimentos complementarios, hasta un mínimo de dos años.

La lactancia materna es la principal fuente de alimentación de los niños y la más importante en los primeros años de vida, ya que esta proporciona al lactante las vitaminas y anticuerpos necesarios para su cuerpo.

Los niños amamantados tienen por lo menos seis veces más posibilidades de supervivencia en los primeros 
meses que los niños no amamantados. La lactancia materna reduce drásticamente las muertes por las infecciones respiratorias agudas y la diarrea, dos importantes causas de mortalidad infantil, así como las muertes por otras enfermedades infecciosas (OMSThe Lancet, 2000). Las consecuencias potenciales de las prácticas óptimas de lactancia materna son especialmente importantes en los países en desarrollo donde se registra una alta carga de enfermedad y un escaso acceso al agua potable y el saneamiento. En cambio, los niños no amamantados de los países industrializados también corren un mayor riesgo de morir: un estudio reciente sobre la mortalidad posneonatal en los Estados Unidos encontró un aumento del $25 \%$ en la mortalidad de los lactantes no amamantados. (UNICEF ,2000)

Confirmando lo anterior en Nicaragua, la alimentación ha venido cambiando los hábitos alimenticios, donde la población considera que es más barato y accesible obtener bebidas carbonatadas (Coca Cola, Pepsi) que frutas para la elaboración de refrescos naturales en la temporada y precios.

UNICEF, (2011) agrega que existen otros factores que inciden como son la ingesta insuficiente de alimentos adecuados, la atención inadecuada y las enfermedades infecciosas, las mujeres son vulnerables a la malnutrición a lo largo del ciclo vital tanto por razones biológicas como sociales.

\section{MATERIALES Y MÉTODOS}

El estudio se llevó en el sistema lagunar PlayitasMoyúa- Tecomapa se localiza en el Departamento de Matagalpa, Municipio de Ciudad Darío, a $66 \mathrm{Km}$ de la Ciudad de Managua y 64 de la ciudad de Matagalpa, las comunidades aledañas al humedal son: San Martín, Puertas Viejas, El Papayal, San Vicente y la Comarca las Playas de Moyúa. Este sistema lagunar forma parte de la cuenca de drenaje del Río Grande de Matagalpa, segundo río más largo de Nicaragua con $368 \mathrm{~km}$ de longitud, y área drenada total de 18309 km (CIRA, 2010).

El estudio se desarrolló en Marzo -Junio del 2015. Los datos fueron recopilados en la época seca, entre los meses de Marzo - Mayo. El estudio es de naturaleza social no experimental con enfoque mixto con variables cualitativas y cuantitativas, de corte transversal, se procesaron los datos en Excel mediante estadística descriptiva, la recopilación secundaria se realizó mediante el instrumento de la encuesta, listado proporcionado por el Consejo Sandinista de Desarrollo Humano (CSDH) y Ministerio de Salud (MINSA).

Con el fin de caracterizar el estado nutricional de las familias a partir de los indicadores del historial clínico de madre: edad del embarazo, peso, talla visita al control prenatal historial clínico del infante: peso al nacer, duración de la lactancia, enfermedades presentadas, peso y talla actual, IMC, tiempo de alimentación, frecuencia de consumo y las condiciones sociales existente en las familias como son: escolaridad, calidad de la vivienda servicios básicos y hacinamiento; la población estaba constituida por 84 familias y la muestra representada por el $50 \%$ de la población total, la tabla 1 representa la distribución de las muestras por comunidad, bajo los criterios de que los padres de familias brindaran las información, al menos un niños menores de cinco años fueran nativos de la comunidad.

Tabla 1: Distribución de la muestra

\begin{tabular}{lrr}
\hline Comunidades & $\begin{array}{c}\text { Familias /niños } \\
\text { (población) }\end{array}$ & (muestra) \\
\hline Moyúa & 12 & 6 \\
Playitas & 20 & 10 \\
Puertas vieja & 34 & 17 \\
Papayal & 18 & 9 \\
\hline Total & 84 & 42 \\
\hline
\end{tabular}

Elaboración Propia 


\section{RESULTADOS Y DISCUSIÓN}

El estudio se realizó en 4 comunidades del municipio de Ciudad Darío, la muestra fue de 42 familias, el $40.47 \%$ de las familias encuestadas forman parte de la comunidad Puertas Viejas, el $23.80 \%$ corresponde a la comunidad Las Playitas, el $14.28 \%$ son de la comunidad de Moyúa, y el $21.42 \%$ pertenece a la comunidad el Papayal.

El estado nutricional de los niños está intrínsecamente relacionado con el crecimiento y desarrollo en las distintas etapas de la vida y debe evaluarse integralmente considerando el crecimiento armónico en relación con la nutrición (PNUD, 2007).

El $86.36 \%$ de las familias realizan los tres tiempos de alimentación, mientras que el $13.63 \%$ no lo realizan. La alimentación facilita el desarrollo físico y mental de las personas, los resultados nos indican que el 13 $\%$ de las familias no tienen alimentos disponibles para los 3 tiempos indicados, esto es una gran desventaja para la producción de proteínas, vitaminas, minerales y aminoácidos que necesita el cuerpo.

Hay diversidad de alimento pero no todas las familias tienen la disponibilidad de consumirlos diariamente, lo que imposibilita que los niños y niñas tengan un mejor desarrollo físico y mental. El consumo de hortalizas, frutas y bebidas naturales son una fuente de vitaminas, siendo importante el consumo de las mismas realizándose de una manera racional.

Los alimentos consumidos con mayor frecuencia por la familia son cereales 55\%, huevos y lácteos $25 \%$ carnes de pollo, res, cerdo, pescado $10 \%$ vegetales $5 \%$ $\mathrm{y}$ frutas $5 \%$.

Según la Encuesta Nacional de Consumo de Alimentos (IV ENCA, 2012), se califica como una dieta suficientemente diversa si hay 10 grupos de alimentos y que esta posiblemente satisfaga las necesidades alimentarias y nutricionales de la familia y si hay de 6 a 9 grupos, la calificación de diversidad es aceptable pero posiblemente la dieta no satisfaga todas las necesidades nutricionales de la familia.

La frecuencia de consumo de un alimento se determina mediante los siguientes rangos : muy frecuente de 6 a 7 días, frecuente 3 a 5 días y poco frecuente 1 a 2 días (IV ENCA, 2012).

La figura 1 representa la edad promedio de la madre en estado de embarazo, según la Atención Integrada a las enfermedades prevalentes de la infancia (AEIPI, MINSA, 2009) en los cuadros de procedimientos de evaluación, clasificación y el trato de la embrazada durante el parto, determina que la edad es un factor muy importante y no poner en peligro al bebe, lo cual considera que la edad promedio debe ser entre los 2035 años, clasificándolo al parto como: bajo riesgo.

Cuyo resultado establecen que la edad promedio de las madres en estado de embarazo oscilan en mayor porcentaje en las comunidades en estudio de menores de 20 años, lo cual coincide con los resultados de Soza \& González, (2014) en la micro cuenca de ApanasJinotega, lo cual indica de que la madre tendrá un parto bajo riesgo.

\section{Gráfico 1: Edad Promedio de la madre en estado de embarazo}

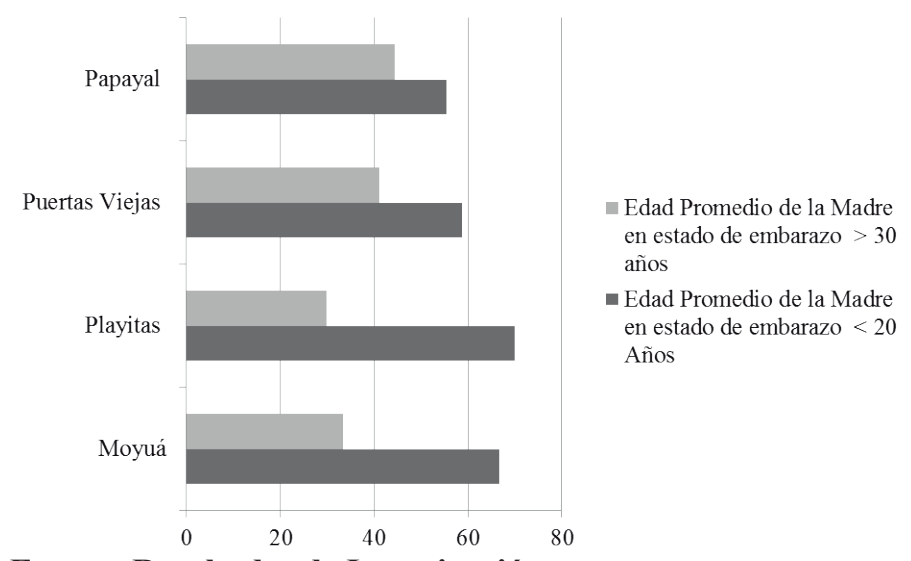

Fuente: Resultados de Investigación 
El gráfico 2 representa el porcentaje de embarazo en riesgo de las madre en comunidades en estudio, 2015, prevaleciendo el embarazo en bajo riesgo tomando los indicadores de edad, peso, talla, y controles prenatales.

\section{Gráfico 2: Porcentaje de embarazo de riesgo de la madre en las comunidades en estudio, 2015}

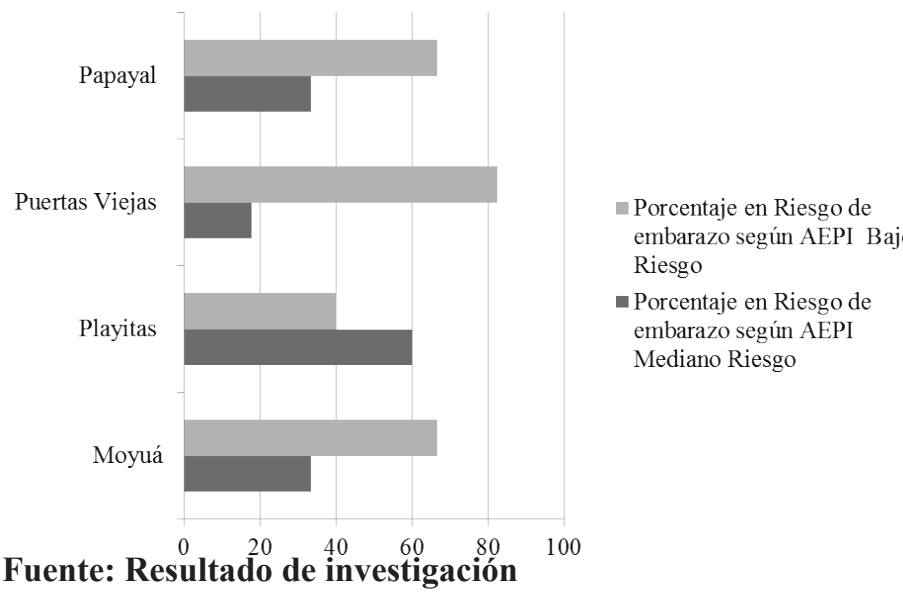

La figura 3 representa la frecuencia con que la mujeres en estado de embarazo realizan visitas el puesto de salud para el debido control tanto de su salud como la del bebe, según el MINSA, (2009) establece que por lo mínimo la madre debe de realizarse el control prenatal $1 \mathrm{vez}$ al mes, podemos observar que en la mayoría las madres asisten al puesto de salud mensualmente, para el debido control prenatal lo cual indica que las madres vigilan el desarrollo de sus hijos, cuyos resultados coincide con los resultados de Soza \& González,(2014). en la micro cuenca de lago de Apanas.

En la actualidad el ministerio de salud de Nicaragua, realiza en los diferentes centro de salud, hospitales charlas educativas con los temas de medidas higiénicas, preparación de los alimentos, vacunación, e importancia de una alimentación sana.

\section{Gráfico 3: Porcentaje de frecuencia de las visitas de} controles prenatales en las comunidades en estudio

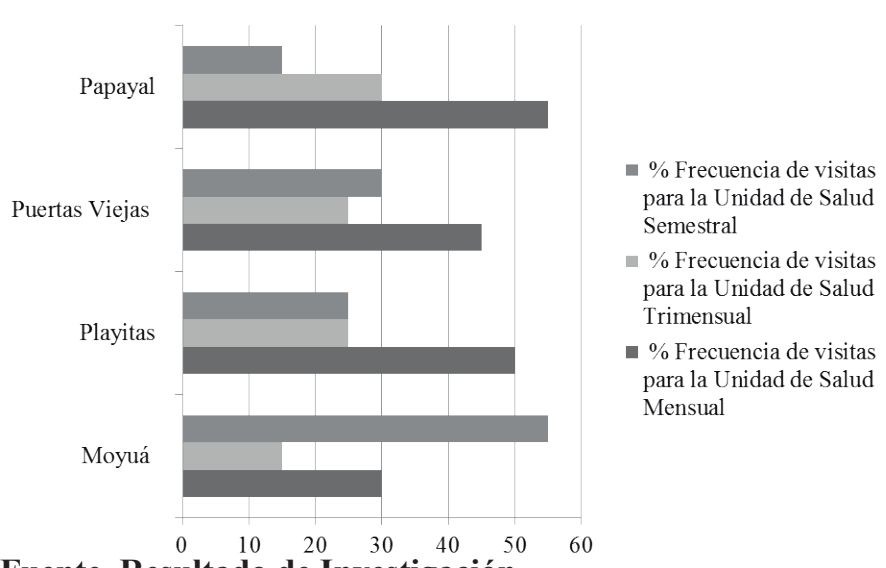

Fuente. Resultado de Investigación

Las enfermedades durante el embarazo en su mayoría son provocados por la inadecuada nutrición de las madres, que el $68.57 \%$ no presentó ningún tipo de enfermedades y solamente un $31.42 \%$ de las mujeres presentaron enfermedades durante el embarazo, como: infección renal, problemas ováricos, etc.

El cuido durante el embarazo es importante ya que de eso depende el bienestar del bebe, además de su buen desarrollo y nacimiento, en los resultados encontrados las enfermedades presentadas son las más comunes, pero estas pueden llegar a provocar riesgos en los embarazos, ya que pueden haber partos prematuros y poco desarrollo del niño.

Según OMS (2010), es el estado de completo bienestar físico, mental, espiritual, emocional y social y no solamente la ausencia de afecciones o enfermedades. El índice de masa corporal (IMC) es un indicador simple de la relación entre el peso y la talla que se utiliza frecuentemente para identificar el grado de sobrepeso y la obesidad en los adultos y niños.

Los datos que muestra la gráfica 4 deduce que el índice de masa corporal (IMC) de los niños y niñas menores de cinco años de edad de las familias del sistema lagunar que de los 33 niños un IMC siendo el mayor de $22.73 \mathrm{Kg} / \mathrm{m} 2$ y el menor de $9 \mathrm{~kg} / \mathrm{m} 2$, la cual según OMS (2004) un IMC normal es de 15.1 a 16.4. 
Gráfico 4 Índice de Masa Corporal (IMC) de los niños menores de cinco años en las comunidades en estudio

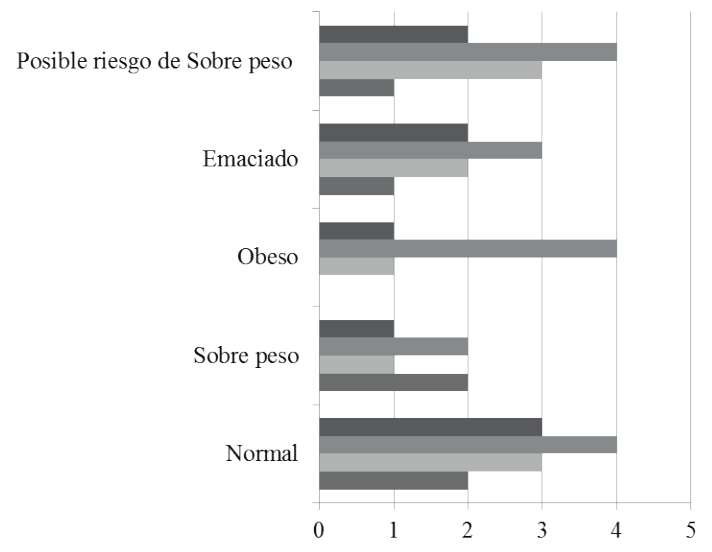

- Papayal - Puertas Viejas Playitas - Moyúa

Fuente: Resultados de Investigación

El grafico 5 representa el porcentaje de peso del bebe al nacer en gramos, donde prevalece los niños obtiene un peso adecuado mayor de 2500 y no son considerados en riesgos, podríamos afirmar que los niños nacen saludables.

El peso promedio de los niños al nacer Según la Atención Integrada a las enfermedades prevalentes de la Infancia (AIEPI), MINSA,( 2009) establece que el recién nacido de alto riesgo los siguientes signos como: peso al nacer $<2000 \mathrm{~g}$ o $>4000$ gr, semanas de gestación $<35$ Temperatura $>35.5 \mathrm{C}^{\circ}$ y otras anomalías de salud que presente la madre y mediano riesgo peso al nacer entre 2000 y $2500 \mathrm{~g}$ edad gestacional entre 3537 semanas y en bajo riesgo un recién nacido vigoroso, Rosado ,llanto fuerte, respiración normal, activo peso $>2500 \mathrm{~g}$ y $<4000 \mathrm{~g}$ con edad gestacional $>37<42$ semanas, los resultados encontrados, en posible riesgo de sobre peso, obesos, sobre peso y emaciado

Esto indica que la mayoría de los niños están en estado de desnutrición, causando mal crecimiento en los niños, retraso en los rendimientos académicos, falta de interés para la recreación y sociabilidad con otros niños de su misma edad.

\section{Gráfico 5 porcentaje de peso al nacer en gramos en} las comunidades en estudio

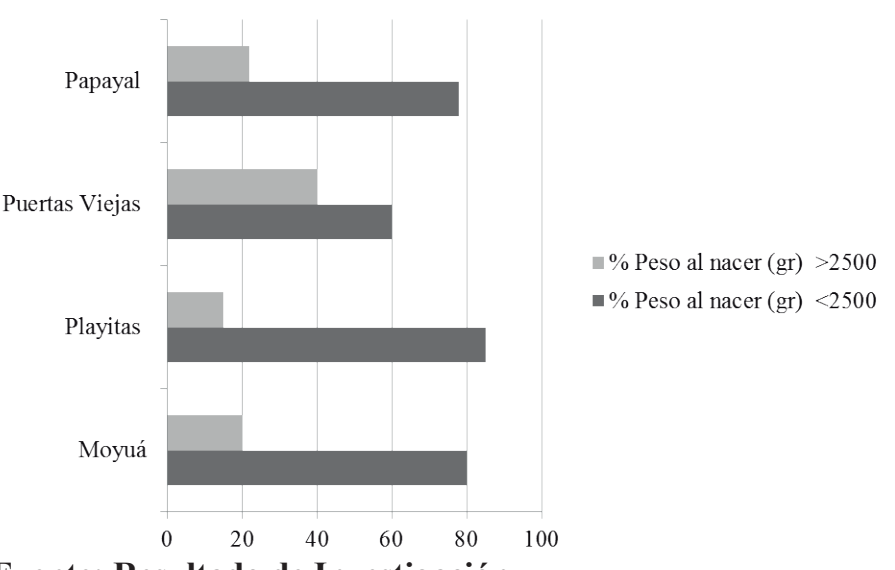

Fuente: Resultado de Investigación

En el caso de América Latina la prevalencia bajo del $22 \%$ entre 1990-2008, También hay noticias alentadoras para el caso de la lactancia materna exclusiva como es el caso de Colombia y Perú en la región donde prevalencia de LME aumento en un 30\%; de $11 \%$ a 47 $\%$ en el caso de Colombia entre (1995-2005) y de un $33 \%$ a $64 \%$ para el caso de Perú (entre 1992-2007). La iniciación temprana de la lactancia materna se encuentra en un $48 \%$ (todas las regiones del mundo excluyendo China, (OPS/OMS,2010).

El Ministerio de Salud de Nicaragua, promueve la lactancia materna, desde la primera media hora después del parto y continuar la lactancia exclusiva hasta los seis meses ya que reúne todos los requerimientos nutricionales con excepción del a vitamina $D$ y Hierro en los lactantes prematuros y con bajo peso del nacimiento y combinarla con alimentación complementaria hasta los dos años (OMS, 2003).

La lactancia materna ha aumentado en 3\% (19932007) de 29 a $31 \%$ (ENDESA, 2007) la misma encuesta evidencia que un $94 \%$ de los niños nacidos en el 2007, al menos una vez recibieron lactancia OPS/ OMS (2010).

El gráfico 6 representa la duración de la lactancia materna en los niños y niñas menores de cinco años en la comunidad de puertas viejas amamanta a los niños 
en periodo de 2 -12 prevaleciendo lo recomendado por el ministerio de salud que es de 3-6 meses.

\section{Gráfico 6: Duración de la Lactancia Materna en las comunidades en estudio}

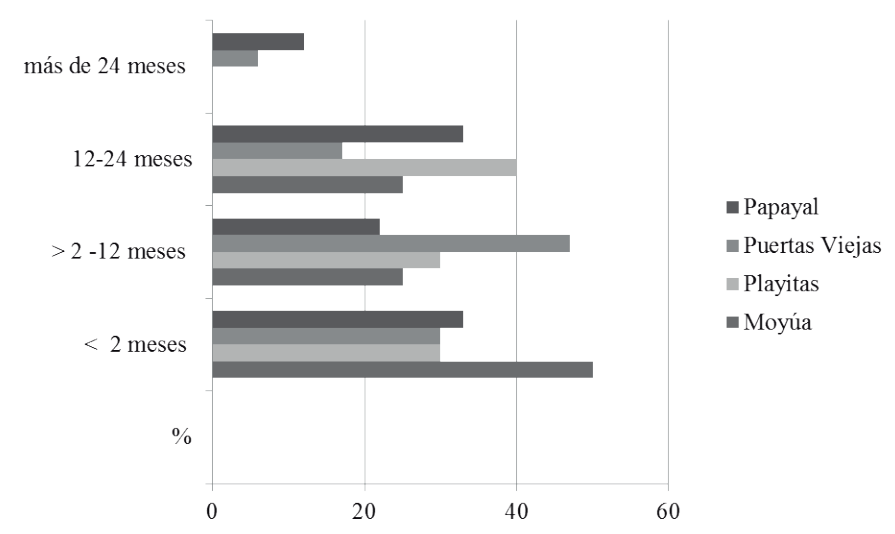

Fuente de Resultados de Investigación

Según PNUD \& OIT (2009), señala que en Nicaragua los principales problemas de vivienda son los materiales con que están construidas generalmente plástico, zin, madera y no satisfacen las necesidades de los servicios básicos necesarios de las familias.

Una vivienda para considerarse como digna debe de cubrir de forma satisfactoria las necesidades básicas sanitarias como: privacidad, comodidad, funcionalidad, ubicación, seguridad en la tenencia y provisión de agua potable y medios de eliminación de excretas y basura (agua de drenajes, alcantarillados) para prevenir enfermedades que alteran el estado de la salud de las personas.

Rojas (2009), plantea que una vivienda digna debe satisfacer simultáneamente 6 requisitos: a) que la vivienda este ocupada por una familia; b) que no tenga más de dos ocupantes por cuarto habitable en el medio urbano y no más de 2.5 en el rural; c) que no esté deteriorada; d) que cuente con agua entubada en su interior; e) que cuente con energía eléctrica.

La aseveración anterior coincide con el planteamiento de INEC, (2005), que la calidad de vivienda es lo que se refiere a la cantidad promedio de personas por vivienda, tipo, tenencia y los servicios básicos (agua, luz, etc.) con que cuentan las mismas.

Las viviendas de las familias en estudio poseen los servicios básicos de agua potable, energía eléctrica, letrinas es de $100 \%$. El estado de las viviendas Buena $59 \%$, Regular $25 \%$ mal estado $16 \%$, material del techo Zinc 94 teja 3, palmera $3 \%$ Paredes Ladrillo $50 \%$ Bloque $25 \%$ piedra cantera $10 \%$ plástico $5 \%$ Madera 6 Zinc 4.material del piso de tierra 57.27, embaldosado 29 y piso de cerámica $13.73 \%$.

Del $100 \%$ de los habitantes de las comunidades del sistema lagunar, el $76.19 \%$ trabajan en la agricultura y el $23.81 \%$ no ejerce ninguna labor, de estas, el $51.22 \%$ trabaja 1 persona dentro del seno familiar, el $34.14 \%$ trabajan 2 personas, el $12.19 \%$ trabajan 3 personas y el $2.43 \%$ trabajan todos los integrantes.

En la mayoría de las familias rurales los padres se ven obligados a emigrar de sus hogares y poder darles una mejor vida económica a sus hijos y familiares. Solamente el $11.62 \%$ de las familias reciben remesas familiares y un $88.37 \%$ no reciben, esto indica que en su mayoría las familias están trabajando en territorio nicaragüense.

El gráfico 7 representa el porcentaje de hacinamiento en las comunidades en estudio Hacinamiento, donde las familias de la comunidad de Papayal existen un $30 \%$ de hacinamiento, es decir es mayor el número de personas que las habitaciones en las viviendas, en lo cual no hay privacidad entre las familias.

Amartya Sen anota de que cada método de medición hay concepciones y las particulares sobre la pobreza y que su uso tiene un interés propio en las áreas de diagnóstico sobre la magnitud del fenómeno y en su forma de enfrentamiento (Sen, 1981.PNUD) con esta orientación, se hace un recuento de los distintos métodos de medición de la pobreza, para establecer la ubicación de la NBI y si concepción de pobreza. A 
partir de allí se exploran las ventajas y limitaciones de uso para fines prácticos de análisis social y para la definición de políticas.

Según Rojas (2009), el termino hacinamiento hace referencia a un estado de cosas lamentables que se caracteriza, por el amontonamiento o acumulación de individuos en un mismo lugar, el cual propósito no se haya físicamente preparado para albergarlos. Es decir que la cantidad de seres humanos que habitan u ocupan un determinado espacio es superior a la capacidad que tal espacio debería y puede contener de acuerdo a los parámetros de comodidad, seguridad e higiene.

\section{Gráfico 7: Porcentaje de hacinamiento de las familias en las comunidades en estudio}

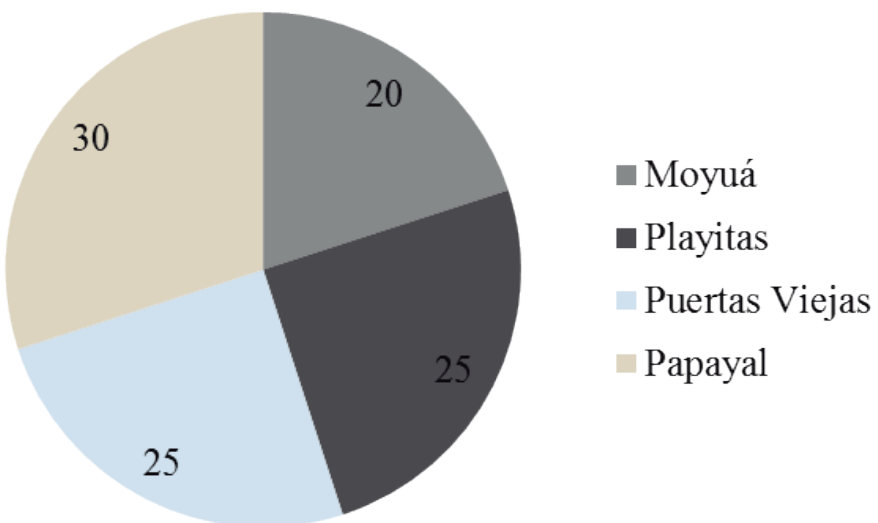

Fuente: Resultados de Investigación

\section{CONCLUSIONES}

Las madres en estado de embarazo de las madres según la edad, control prenatal, peso y talla es considerados embarazo en bajo riesgo; no poniendo en riesgo la salud del bebe.

Los niños y niñas menores de cinco años de edad se clasifican según los patrones de crecimientos de la OMS, AEIPI, (2009) con el índice de masa corporal (IMC) desde posible riesgo, obeso, sobre peso Emaciado lo indica que los alimentos consumidos no están solventando las necesidades fisiológicas de los infantes; a pesar que nacen con el peso adecuado, considerado como saludables, bajo atención medica de hospitales en su mayoría.

La disponibilidad económica de las familias se dedica a la agricultura, albañilería, y la madre de auxiliares del hogar, los jefes de familia se ven obligados a emigrar a otras comunidades o municipios.

Las familias poseen los servicios básicos de energía, agua potable y letrinificacion, el estado de la vivienda consideras en Bueno y regular estado.

\section{REFERENCIAS BIBLIOGRÁFICAS}

Amartya Sen, (2000) Desarrollo y Libertad, Buenos Aires editorial Planeta.

Andreu, A. (2012). Nuevos trastornos de la conducta alimentaria. Índice de Masa Corporal. Francia.

AIEPI, (2009) Atención Integral a las enfermedades prevalentes de la infancia Cuadro de Procedimientos para la atención integral de la niñez tercer edición NWS 141-0451-2008, p 52 Génesis

Bernal, I.J.R. (2010) Inseguridad alimentaria y hambre en niños:Diseño y validacion de instrumento para estudio Universidad simon Bolivar Venzuela.

Casanueva, E., Kaufer, M.,Pérez y Arrollo, P. (2002). Nutrición Médica. 2da edicion. México: Médica panamericana.

CEPAL (2009). Panorma social de America Latina. Comision económica de las Naciones Unidas para América latina y el Caribe. Santiago de Chile.

CIRA (2010) Centro de Investigacion de Recursos Aguaticos."Plan de gestion y Desarrollo Integral en la sub cuencas de las playitas,Moyua y Tecomapas dela cuenca del rios grande de Matagalpa ,municipio de Ciudad Dario.

Delgado. (2005)"La seguridad alimentaria y nutricional, Un enfoque Integral, Síntesis de los desafíos y experiencia en Centroamérica" INCAP/ OPS, Guatemala

ENCA,2012 Encuesta nacionall de salud y Nutricion,2012 ensanut inspmx/informe/ 
ENsanut2012.

ENDESA (2006) Encuesta Nicaragüense de Demografia y Salud web; www.inide.gob.ni

FAO,(2006) Estado de la seguridad alimentaria y nutricional en Nicaragua. "hacia la elaboracion de una estrategia de asitencia tecnica de la FAoen apoyo a la implementacion de la iniciativa de America Latina y el Carib sin hambre, Guatemala.

FAO, (2007). Programa especial para la Seguridad Alimentaria y nutricional. En web:http://WWW. fao.org/docrep/006/AC828S00.HTM

OMS, Organización Mundial de la Salud (1979). Condición de salud del niño en las Américas. Ginebra

OMS, (2003)Ambientes saludables para los niños www.ho.int/word-health-day/previos/2003/es/.

OMS, (2005) Organización Mundial de la salud, Patrones de crecimiento y Desarrollo Infantil del niño y la niña, Cursos de Capacitación sobre la evaluación del crecimiento de la niñez Editorial El manual moderno S.A de CV Colombia, 2014 tercera edición DR ISBN 978-607-448-380 miembro de la cámara Nacional de la Industria Editorial Mexicana, Reg. Núm. 39.

OMS/MINSA/AIEPI,(2009) Atencion Integral a las enfermedades prevalentes de la infancia Cuadro de Porcedimientos para la atencion integral de la niñez tercer edicion NWS 141-0451-2008 ,p 52 Genesis.

OMS, (2010) Informe sobre Salud en el mundo"La financiando de los sitema de salud,Berling alemania.
Soza \& González, (2014) “Caracterización agroecológica de tres comunidades del lago de Apanás para optar al título de ingeniero agrónomo, Facultad Multidisciplinaria, Matagalpa, UNAN Managua.

PNUD,(2007) Informe Sobre Desarrollo Humano 2007-2008 hdr.undp.org/Siles/default/file/ hdr_20072008_summery Spanish PDF.

PNUD, P, \& OIT /OMS, (2009). Trabajo y Familia: Hacia nuevas formas de conciliación con responsabilidades. México.

PNUD, (2012). “Guatemala un Pais de Oportunidades para la Juventud.Informe Nacional de Desarrollo Humano 2011/2012. Guatemala:p 330. Editorial Don Quijote ISBN: 978-9929-606-00-5.

Rojas J,E,M (2009). Cooperativismo y desarrollo humano "Una propuesta metodologica para su medicion. Primera edicion. Editorial Apante p209 ISBN 978-99924-0885-8.

Rojas, J, E, M (2013). Enfoque, metodología y experiencias Desarrollo Rural. Territorial. Nicaragua Bolonia Printing. Managua, Nicaragua.

UNICEF, (2000) www.unicef.org/Spanish/ nutriciónIindex_24824 htm.

UNICEF, (2011) "La desnutrición Infantil causas, consecuencias y estrategias para su prevención y tratamiento España

Wisbaum, W. (2011). Desnutricion Infantil, Consecuencias y Estrategias para su prevencion y tratamiento. España: UNICEF. 